\title{
Robust Face Detection integrating Novel Skin Color Matching under Variant Illumination Conditions
}

\author{
Asif Anjum Akash', M. A. H. Akhand ${ }^{1}$, N. Siddique ${ }^{2}$ \\ ${ }^{1}$ Dept. of CSE, Khulna University of Engineering \& Technology, Khulna, Bangladesh \\ ${ }^{2}$ School of Computing, Engineering and Intelligent Systems, Ulster University, United Kingdom \\ Email: ${ }^{1}$ \{akash.kuet23@ kuet.ac.bd, akhand@cse.kuet.ac.bd $\} ;{ }^{2}$ nh.siddique@ulster.ac.uk
}

Received: 16 July 2020; Accepted: 11 September 2020; Published: 08 April 2021

\begin{abstract}
Integration of skin color property in face detection algorithm is a recent trend to improve accuracy. The existing skin color matching techniques are illumination condition dependent, which directly impacts the face detection algorithm. In this study, a novel illumination condition invariant skin color matching method is proposed which is a composite of two rules to balance the high and low intensity facial images by individual rule. The proposed skin color matching method is incorporated into Haar Feature based Face Detection (HFFD) algorithm for face detection and is verified on a large set of images having variety of skin colors and also varying illumination intensities. Experimental results reveal the effectiveness and robustness of the proposed method outperforming other existing methods.
\end{abstract}

Index Terms: Face detection, Haar feature, skin color matching, illumination

\section{Introduction}

A face detection method extracts features from a facial image or a video stream disregarding background images. Face detection is a non-contact method applied to authentication and recognition systems [1]. Face detection is useful in various applications such as online job application to verify real face, embedded face detection system in smartphone for auto focusing, smart home users' face detection, biometric system, suspect detection and crime deterrent.

Face detection is a challenging task and different techniques are investigated in several years. Human vision system is amazing in detecting face easily but it is very challenging in computer vision and image processing as it depends on quality of image, illumination, lighting conditions, facial expressions, face sizes, occlusions, face position, face rotation angle and cluttered background etc. Therefore accuracy is an important issue here and several researches $[2,3]$ are found to improve accuracy of face detection by different ways. Among different technologies, multilayer neural networks [4-9], deep learning [10], support vector machine (SVM) [11], particle swarm optimization (PSO) [12], template matching [13,14], and kernel probability map [15] are popular methods for face detection. There exists also example based [16], component based [17] and many more [18-21] face detection systems. But the most popular and widely used face detection systems are feature matching based face detection systems [1, 22-26].

Several studies [27-30] investigated the current research trends of face detection systems; in general, they divided into image-based and feature-based approaches [30]. Among the feature-based face detection approaches, Haar feature based face detection (HFFD) system is the most widely used method [1,22]. Viola and Jones [22] uses classical face detection framework based on the boosting strategy but fails detecting faces from different angles and of variable resolution. Among image-based face detection approaches, neural network is the most popular method [31]. Kang and Park [32], Bianchini et al. [33] and El-Bakry [34] reported extensions of neural network based methods improving the performance of human face detection in different lighting conditions and cluttered background. Image-based face detection methods apply window scanning technique to locate face in the image leading to high computational time.

The existing face detection systems including HFFD very often detect a region as a face which is eventually not a face. To counteract such false detections, skin color properties in different color space are incorporated into face detection methods. A number of studies incorporating skin color [1,35-47] are found to have improved the detection accuracy. It is obvious that the existing skin color matching techniques are illumination condition dependent, which directly impacts the face detection method. A method performs well in a particular illumination condition (e.g., low light intensity) but at the same time the same method performs badly in other illumination conditions (e.g., high light intensity). Therefore, face detection with existing skin color matching techniques is not robust and performance degrades when applied to images with different illumination conditions. An illumination invariant skin color detection 
method is deemed necessary for better face detection.

The aim of this research is to devise a robust face detection system integrating skin color matching that will perform reliably under different illumination conditions. It is very challenging to formulate a rule which is capable of detecting human skin color for a wide range of color space and for a broad range of illumination conditions. In pursuit of this goal, a novel skin color matching method is proposed which is a composite of two rules to balance the high and low intensity facial images by individual rule. The proposed hybrid skin color matching with HFFD verified on a large set of images with varying illumination conditions and varying skin colors is revealed to be a robust face detection system.

The initial result of this research was published in a conference and this paper is the complete presentation of the study with extensive improvement of the conference paper [48]. The proposed algorithm of this study is an improved version of conference one and is presented with a flowchart for better understanding. The novelty of the method is clarified in comparison with the existing methods. Moreover, extensive analysis has been carried out on three benchmark datasets and performance compared with existing methods.

The remainder of this paper is structured as follows. Section II investigates several existing skin color detection methods. Section III describes the proposed robust hybrid face detection method with the proposed novel skin color matching. Section IV is for experimental studies where the proficiency of the proposed method is compared with traditional methods. Section V concludes the paper with few remarks.

\section{Related Works}

There are two ways to improve accuracy of face detection. One is the increasing true face detection rate (increasing true positive rate) and the other is the decreasing false detection rate (reducing false positive). Reducing the wrong detections integrating human skin color matching is well studied to improve the accuracy of face detection. There are many human skin color detection rules proposed in the literature. However, the traditional human skin color detection method $[39,49-51]$ has limitations because of skin color is subject to illumination condition and applicable to certain domains. Therefore, to overcome the limitations and to improve accuracy, a few hybrid skin color detection methods [51-54] combining two or more traditional methods have been reported in the literature. This section first investigates the strength and limitations of the different popular traditional skin color detection methods incorporating them into HFFD followed by investigation of the hybrid skin color detection methods.

\section{A. Analysis of Traditional Skin Color Detection Methods}

$\mathrm{RGB}$ and $\mathrm{YCbCr}$ color spaces are popular for skin color detection in the literature. RGB color model is very simple where R, G and B represent the value of red, green and blue components of a pixel, respectively. The range of R, $\mathrm{G}$ and $\mathrm{B}$ components is $[0,255]$. On the other hand, $\mathrm{YCbCr}$ color space is the transformation of RGB color space. The four widely used traditional skin color detection methods and their effectiveness on illumination conditions are described below.

\section{i) RGB Space with Fixed Values}

RGB is the basic color space and widely used method for skin color detection [39], [49]. In this method, the pixel representing the skin color holds the following:

$$
\begin{aligned}
& (R>95) \wedge(G>40) \wedge(B>20) \wedge \\
& {[(\max (R, G, B)-\min (R, G, B))>15] \wedge} \\
& (|R-G|>15) \wedge(R>G) \wedge(R>B)
\end{aligned}
$$

In this study, the method described by Eq. (1) is investigated using images with different illumination intensity. It is found that this method works quite well for the images with bright illumination condition but suffers from the images with dark illumination condition. The individual RGB values of a dark skin is less than a brighter skin; thus, the Eq. (1) with fixed RGB values for detecting bright skin color will not be able to detect dark skin color.

\section{ii) Normalized RGB Ratio}

Gomez and Morales [50] employed the normalized RGB method where a pixel is considered human skin color if the following holds:

$$
\left(\frac{r}{g}>1.185\right) \wedge\left(\frac{r * b}{(r+g+b)^{2}}>0.107\right) \wedge\left(\frac{r * g}{(r+g+b)^{2}}>0.112\right)
$$

where $\mathrm{r}, \mathrm{g}$ and $\mathrm{b}$ are normalized forms defined by 


$$
\left[\begin{array}{l}
r \\
g \\
b
\end{array}\right]=\left[\begin{array}{ccc}
\frac{1}{R+G+B} & 0 & 0 \\
0 & \frac{1}{R+G+B} & 0 \\
0 & 0 & \frac{1}{R+G+B}
\end{array}\right]\left[\begin{array}{l}
R \\
G \\
B
\end{array}\right]
$$

This method described by Eq. (2) differs from the previous method by transforming the fixed RGB values with their normalized ratios in color matching conditions. Authors mentioned that this method worked well for all types of illumination conditions. But our investigation shows that this method performs average for all illumination conditions and different skin colors; and it fails to detect face under low light condition or dark face.

\section{iii) RGB Ratio}

Recently, Asif et al. [1] investigated the simplest way to detect human skin color; a pixel will be human skin color if its $R, G$ and $B$ values follows:

$$
(R>G>B)
$$

The authors found the method worked much better than other methods in low light and for dark skin colors while tested on a diverse skin colors and a wide range of illumination conditions. However, the method shows slightly badly in comparison with the method in (i) in the case for bright illumination.

\section{iv) $\mathrm{YCbCr}$ Space}

Kukharev and Nowosielski [51] investigated YCbCr color space for human skin color detection. In the model, a pixel will be human skin color if it follows:

$$
(Y>80) \wedge(85<C b<135) \wedge(135<C r<180)
$$

In Eq. (4), Y (luminance), $\mathrm{Cb}$ (chrominance blue) and $\mathrm{Cr}$ (chrominance red) are the chrominance information from RGB components defined by

$$
\left[\begin{array}{c}
Y \\
C b \\
C r
\end{array}\right]=\left[\begin{array}{ccc}
0.257 & 0.504 & 0.098 \\
-0.148 & -0.291 & 0.439 \\
0.439 & -0.368 & -0.071
\end{array}\right]\left[\begin{array}{l}
R \\
G \\
B
\end{array}\right]+\left[\begin{array}{c}
16 \\
128 \\
128
\end{array}\right]
$$

The model in Eq. (4) is developed investigating only 25 instances of different skin color without black skin [51]. In our investigation, the model failed to detect dark skinned face and face under low light. However, the model is found slightly better than normalized RGB model in (ii) but slightly worse in comparison with fixed RGB model in (i) where light condition is good and skin color is not black.

\section{B. Analysis of Hybrid Skin Color Detection Methods}

Several hybrid skin color detection methods are proposed combining two or more traditional skin color detection methods discussed in Section II(A). Three popular methods along with their main limitations are described below.

\section{i) Hybrid Method 1: $\mathrm{RGB}+\mathrm{YCbCr}$}

Georgy and Nowosielski [51] used this hybrid skin color detection method which is a combination of a set of RGB rules and a set of YCbCr rules. In the rule, a pixel that holds human skin color property will satisfy the following rules:

$$
\begin{aligned}
& (R>G) \wedge \\
& {\left[\begin{array}{l}
\text { if }(G>B) \text { then }((12 \times G-7 \times B) \leq(5 \times R)) \\
\text { else }((12 \times B-7 \times G) \leq(5 \times R))
\end{array}\right] \vee} \\
& {[(135<C r<180) \wedge(85<C b<135) \wedge(Y>80)]}
\end{aligned}
$$

This method is shown to outperform face detection with traditional skin color detection methods discussed in Section II(A) but true negative (i.e., detection of non-face area as face) is relatively high. This method is implemented in this study with HFFD to measure its performance. 
ii) Hybrid Method 2: Red + HSL

Berbar [52] proposed a skin color detection method which used four components: hue (H), saturation (S), lightness (L) and red value (R). Color of an area or a pixel is described by hue; intensity of the color is described by saturation; and brightness is described by lightness. A pixel having position $p(i, j)$ holds human skin color property if:

$$
\begin{aligned}
& H[i, j]<24 \text { and } R[i, j]>125 \text { and } S[i, j]>20 \text { and } L[i, j]>80 \text { or } \\
& H[i, j]>185 \text { and } R[i, j]>155 \text { and } S[i, j]>20 \text { and } L(i, j)>114
\end{aligned}
$$

This method is shown to outperform Hybrid Method 1 for a benchmark dataset [52]. Still the method shows true negative high.

iii) Hybrid Method 3: $R G B+Y C b C r+H S V$

Yadav and Nain [53] investigated a more complicated hybrid skin color detection method. Skin color filtering formula of this method is:

$$
\begin{gathered}
{[(R>95) \cap(G>40) \cap(B>20) \cap(\max (V)-\min (V)>15)} \\
\cap(\operatorname{abs}(R-G) \geq 15) \cap(R>G) \cap(R>B)] \\
\quad \text { and } \\
{[(Y \geq 80) \cap(10 \leq C r \leq 45) \cap(85 \leq C b \leq 135)]} \\
\quad \text { and } \\
{[(0<H<35) \cup(325<H<360) \cap(0.2<S<0.6) \cap(V \geq 20)]}
\end{gathered}
$$

where $V=[R, G, B]$.

Authors tested the method on different datasets and it is shown to outperform other methods in face detection showing minimum true negative.

\section{Observation from Existing Human Skin Color Methods in Face Detection Application}

Traditional skin color detection methods have their own advantages depending on the particular conditions. Such a method incorporation into a face detection method (e.g., HFFD) is not very effective for images with different illumination conditions, especially in face detection applications as it does not improve the accuracy significantly. On the other hand, existing hybrid skin color detection methods performed better in face detection as a hybrid method of Section II(B) considers two or more the traditional human skin color detection methods of Section II(A) with relational operators "and" and "or" [46-49]. We have carefully observed that combining individual methods with "and" operator increases true negative, i.e., discards actual faces in increasing number. On the other hand, the use of "or" increases false positives, i.e., identifies more patterns as faces those are not human faces. Besides these, different illumination conditions may exist in images in a dataset and even in the same image at different portions. Thus, the choice of methods based on illumination is eventually not useful when the illumination condition is not predefined. A robust face detection method that might work well for different illumination conditions is an open research challenge. The challenge to devise a novel skin color detection method for robust face detection is carefully addressed and tackled in this study.

\section{Robust Face Detection using Novel Skin Color Detection Method}

To develop a robust face detection method, existing skin color detection methods with popular face detection method HFFD are investigated as preliminary work of the present study. Fixed RGB values (i.e., Eq. (1)) with standard HFFD outperforms other methods (e.g., [51]) when skin color is white or illumination condition is good; but it suffers from dark skin color or low light condition. On the other hand, RGB ratio (i.e., Eq. (3)) performs much better compared to other methods (e.g., [51]) for dark skin or low light condition. Combining the complementary strengths of the two methods, a novel skin color detection method is proposed and investigated. There are two major steps in the proposed method.

- Preliminary face detection is performed applying standard feature matching using HAAR features.

- Filtering is used to detect real faces applying the proposed novel skin color matching.

Human skin color matching will discard false detections which are primarily detected as human faces in the first step. The following subsections describe the steps briefly. 


\section{A. Preliminary Face Detection by Feature Matching}

A common feature of human face is the appropriate placement of eyes, nose and mouth. Face detection with HAAR features is very popular [22]. HAAR features are rectangular featured areas which describe parts of an image object as a face. Fig. 1 shows only three HAAR features representing the eye area, the nose area and the mouth area of a human face among a lot of features. The details of feature selection and HAAR feature based face detection (HFFD) process can be found in $[1,22]$.

The feature matching-based approach returns an area as a face where it matches eye, nose and mouth features. In many cases, the method returns an area as a face which is not a real human face but it has features like a face with eye, nose and mouth. Such false positive decreases overall performance of a face detection method. Elimination of such false positive improves the face detection accuracy.

\section{B. Elimination of False Faces by the Proposed Novel Skin Color Matching Method}

The outcome of HFFD, the preliminary face detection step explained in previous section, might contain false positives face regions. A novel human skin color matching formula, developed based on composite rules, is applied to those regions to discard false positives. The proposed skin color matching checks each detected facial regions individually. At first, average light intensity of a given facial region will be calculated and then appropriate color filter rule from two developed rules will be applied for final skin color filtering based on the average light intensity. Average light intensity of a detected facial region is an important issue in the proposed method for appropriate skin color matching rule selection. Light intensity $\left(L\left(p^{i}\right)\right)$ of $i$ th pixel $p^{i}$ is found by the Eq. (9)[55].

$$
L\left(p^{i}\right)=\frac{\operatorname{MAX}\left[p^{i}(\mathrm{R}, \mathrm{G}, \mathrm{B})\right]+\operatorname{MIN}\left[p^{i}(\mathrm{R}, \mathrm{G}, \mathrm{B})\right]}{2},
$$

where $\operatorname{MAX}\left[p^{i}(\mathrm{R}, \mathrm{G}, \mathrm{B})\right]$ returns the maximum value among $(\mathrm{R}, \mathrm{G}, \mathrm{B})$ of $i$ th pixel $p^{i}$ and $\operatorname{MIN}\left[p^{i}(\mathrm{R}, \mathrm{G}, \mathrm{B})\right]$ returns the minimum value among $(\mathrm{R}, \mathrm{G}, \mathrm{B})$ of $p^{i}$.

Average light intensity $L^{a v}$ of a region having n pixels is:

$$
L^{a v}=\frac{\sum_{i=0}^{n} L(p i)}{n}
$$

The two rules, from which one is selected, based on calculated $L^{a v}$ are shown in Eq. (11) and Eq. (12). Rule 1 is the RGB with fixed values explained in Section II(A)(i) as Eq. (1). Rule 2 is a modified version of RGB ratio explained in Section II(A)(iii) as Eq. (3). This modification of RGB ratio makes the detection process more robust, which has been incorporated into the model after verifying it on various complex images. If $L^{a v}$ is high with respect to defined threshold value $L^{\theta}$, Rule 1 will be used as color filter; otherwise Rule 2 will be used.

$\underline{\text { Rule } 1 \text { (RGB with fixed values): }}$

$$
\begin{aligned}
& (R>95) \wedge(G>40) \wedge(B>20) \wedge \\
& {[(\max (R, G, B)-\min (R, G, B))>15] \wedge} \\
& (|R-G|>15) \wedge(R>G) \wedge(R>B)
\end{aligned}
$$

$\underline{\text { Rule } 2 \text { (modified RGB ratio): }}$

$$
(R>G>\max [(B-10), 0])
$$

The composite human color detection development is the main contribution of the work. To make the face detection robust, the two rules are selected very carefully to tackle wide range of illumination conditions (i.e., very low intensity to high intensity) into images. It is already discussed that the method of Rule 1 is the best suited method for the images with bright illumination condition. In contrary, Rule 2 is appropriate for low illumination condition which is a modified version of RGB ratio method of Eq. 3 with special condition of blue (B) component. B value is reduced by 10 in ratio calculation to bring higher true positives of the rule. It is observed that in many facial images especially in the low light images, green value and blue value is very close even sometimes blue value is a little bit higher than the green value. In such a case, simple RGB ratio fails to satisfy the skin color condition. To tackle the matter, blue value is lowered by 10 and the value is selected analyzing different types of images. Finally, for low intensity facial images, if a human color pixel doesn't satisfy RGB ratio (i.e., R>G>B) it may satisfy Rule 2 and can be truly classified a human color pixel. 


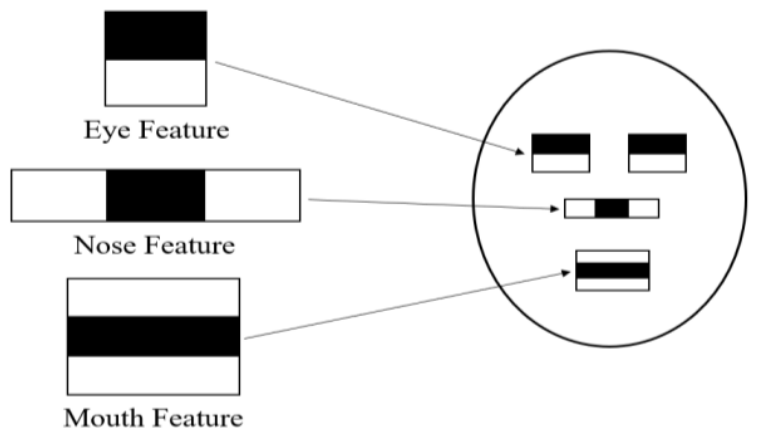

Fig. 1. Detecting human face with rectangular features of eye, nose and mouth

An appropriate threshold value (called $L^{\theta}$ ) for light intensity is an important matter for appropriate rule selection. $L^{\theta}$ is tuned very carefully for the best performance; tuning result will be presented in the experimental analysis section. From this experiment, the best result is found when $L^{\theta}$ is set to 35. Thus, Rule 1 is applied for $L^{a v}>35$; otherwise, Rule 2 is considered as color filter.
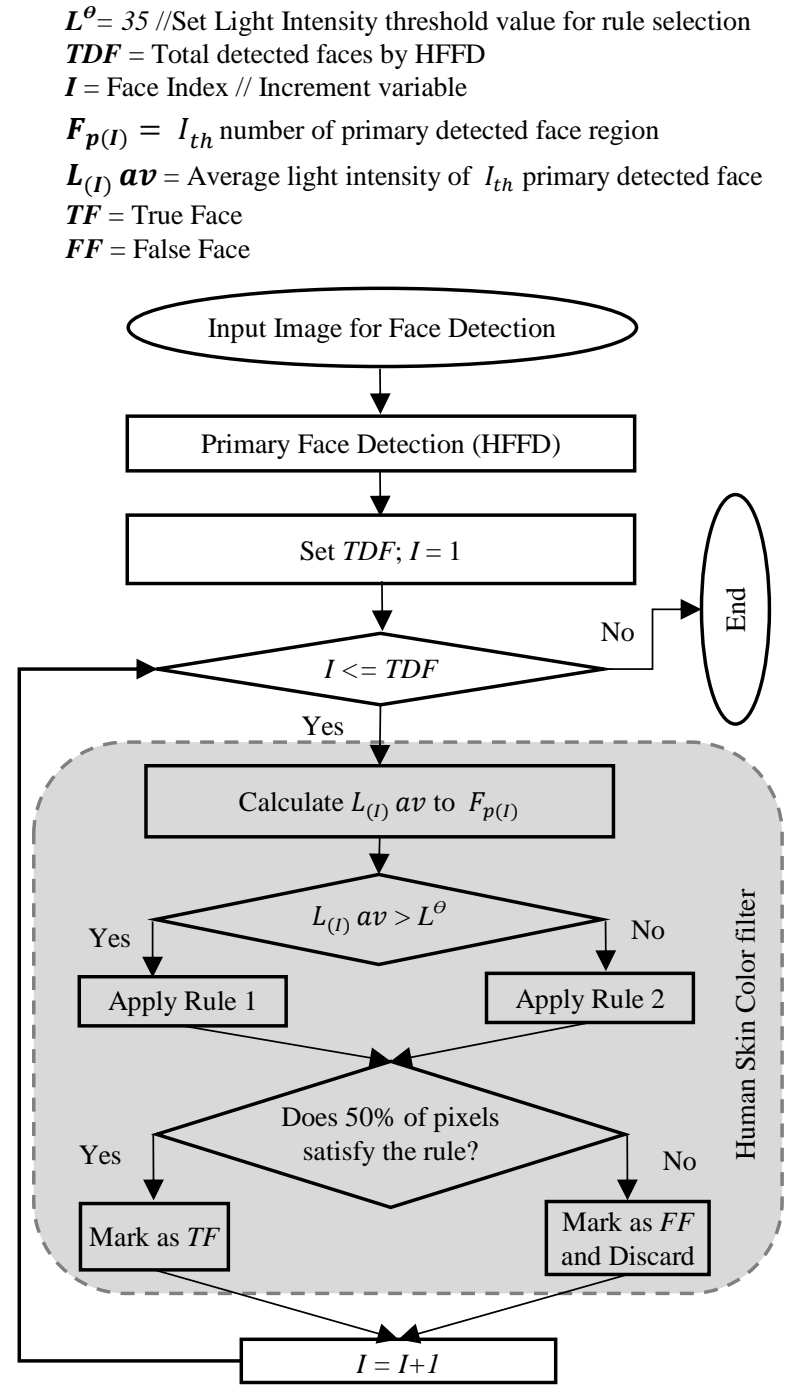

Fig. 2. Flowchart of the proposed face detection algorithm

The proposed skin color matching is performed individually on each preliminary detected facial area. Detected faces may be different in sizes and computational time of the pixel wise color matching for a particular face depends on its size. Each and every pixel consideration for color matching is time consuming to check a facial region and it is obviously too high for a large facial area. To reduce computational cost, proposed method considers pixels for color 
matching at 5-pixel interval throughout the facial area. The facial area is considered as a true human face if more than $50 \%$ of the selected pixels satisfy the color matching criteria; otherwise, the area discarded as a false face. It is worth mentionable that all the pixels of a facial area do not satisfy human skin color due to the existence of non-skin parts like beard, eyes, mustache etc. Therefore, $50 \%$ satisfaction is justified and selected based on observations on several images.

Figure 2 shows the flowchart of the proposed face detection method. At first standard HFFD returns preliminary areas for faces. An image may contain multiple faces and number of total detected faces is denoted as TDF. For all the preliminary detected faces average light intensity $\left(L^{a v}\right)$ is calculated and checked with $L^{\theta}$. For a face, Rule 1 is applied for skin color matching if $L^{a v}>L^{\theta}$; otherwise Rule 2 is applied. If detected facial area satisfy the color matching the face is consider as true face $(T F)$ otherwise mark the area as false face $(F F)$. Finally, all the false faces are discarded and only true positive faces are returned as the proposed method's outcome. Verification of individual faces (i.e., facial areas) using proposed human skin color matching is the significant portion of the algorithm and is shown by the shaded box in the flowchart.

\section{Novelty of the Proposed Method}

The novelty of proposed method is that its filtering process is according to illumination intensity. In this study, it is found that the strength and weakness of every human skin color filtering method depend on illumination intensity. Proposed method selects two best suited methods, Rule 1 in Eq. (11) and Rule 2 in Eq. (12) from the existing methods with appropriate modification for face filtering purpose. Rule 1 is very good for bright scenario and for bright skinned face and Rule 2 is very good for low light condition and for dark skinned face. The proposed method achieved the strengths of the two methods which help increasing the accuracy significantly and detecting faces of variety of races. The experimental results also show that the proposed method is capable of detecting faces of from images with low illumination intensity.

\section{Experimental Analysis}

This section investigates the effectiveness of the proposed face detection method on different facial image datasets as well as combination of those datasets. The proficiency of the proposed method is also identified comparing with four traditional and three hybrid human skin color-based face detection methods. The standard HFFD without skin color detection is also considered for better realization of skin color integration. Four traditional skin color detection methods with HFFD methods will be termed in the rest of the paper as follows.

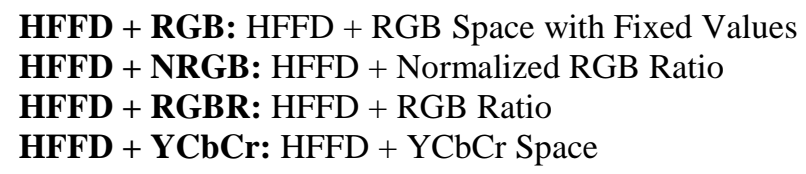

On the other hand, the hybrid methods of Section II(B) will be identified as Method 1 (RGB+ YCbCr)[50], Method 2 (Red + HSL)[51] and Method 3 (RGB + YCbCr +HSV)[52]. The following subsections describe experimental setup, benchmark datasets, illumination intensity threshold value selection, and the experimental results and comparison.

\section{A. Performance Measure and Experimental Settings}

Performance measurements of face detection methods are calculated by the terms - True Positive (TP), True Negative (TN) and False Positive (FP). TP means the detector output is positive (detects a face) and postulates a true face, TN means the detector output is negative (does not detect a face) but postulates a true face and FP means the detector output is positive (detects a face) and postulates a false face (no existence of face). It is notable that in such case there is no false negative (FN), i.e., detector output is negative and postulates a false face. That is why we can assume that $\mathrm{FN}=0$ for this case. That is why, accuracy of a system is measured by the following formula[56].

$$
\text { Accuracy }=\frac{\mathrm{TP}}{\mathrm{TP}+\mathrm{FP}+\mathrm{TN}}
$$

The widely used OpenCV library is considered for implementing HFFD and other methods. OpenCV uses the cascade classifier named lbpcascade_frontalface for frontal face detection. All the experiments were implemented in Java on NetBeans 7.4 IDE. Experiments have been conducted on a desktop computer Dell OptiPlex 3050 (Intel (R) Core i5 CPU $3.40 \mathrm{GHz}, 4 \mathrm{~GB}$ RAM) with 64-bit Windows 8 Professional environment. 


\section{B. Facial Image Data}

Four popular image datasets Caltech[57], Put Face[58], Bao[59] and Muct[60] are used in this study. The Caltech dataset comprises 450 images of size $896 \times 592$ containing 466 faces. On the other hand, Put Face dataset has ten frontal face datasets each containing around 10000 images. Each image contains one face. Images of the datasets have around similar illumination conditions and properties. Therefore, only the dataset 'Images_031_040' is used in this study which has a total of 1028 images containing 1028 faces and size of each image is $2048 \times 1536$ pixels. Bao dataset contains two sets of images. The set having 221 different sized images (with multiple faces containing 1206 faces) is used in this study. Among the 1206 faces, 1152 are frontal faces and rest of the faces are partial faces having different orientation angle. Another set of Bao dataset contains 149 single face images of different sizes, among them the frontal face images are 122. Muct dataset contains five sets of images. Each set contains 751 images of same type having different rotation angle of face. The set named 'muct-a-jpg-v1' is used in this study which has only frontal face of size $640 \times 480$ pixels. Many of the images have artificial bluish lighting effect. Therefore, many of them have lost original human color property. A custom dataset of more challenging images especially having black skinned faces has been created consisting of 20 images of different resolutions and of 310 faces. The images are collected from internet. The images are of different illuminations to the different parts and of different skinned color faces.

\section{Light Intensity Threshold Value (LO) Selection}

Selection of $L^{\theta}$ is an important issue to get the best performance of the proposed method. It is known that $L^{\theta}=35$ is the best suited setting. Experiments have been conducted on all the images of Caltech, Put Face and Custom datasets as they cover a good variety in images. Fig. 3 shows the accuracy of the proposed method on the datasets varying the value of $L^{\theta}$ from 0 to 100 with an interval of 15 . Put Face dataset is almost invariant to the respective threshold values, however, the maximum accuracy of $95.55 \%$ is achieved between 0 and 55 . It is because the dataset contains clean background images with very high value of $L^{a v}$ (i.e., average illumination intensity) and normally more than 55. For Caltech dataset, the highest accuracy of $91.93 \%$ is obtained at $L^{\theta}=35$ and the accuracy becomes slightly lower for $L^{\theta}>$ 35. On the other hand, the performance is very sensitive to lower values of $L^{\theta}$ (e.g., below 30) on Custom dataset; the accuracy is around $60 \%$ for $L^{\theta}<25$. For this dataset, the best accuracy of $78.19 \%$ is obtained at $L^{\Theta}=35$. The custom dataset has darker images. Therefore, it is likely that Rule 1 (i.e., HFFD + RGB) will not work well on this. Setting a low value for $L^{\theta}$ means filtering most of the images by Rule 1 . Thus, the accuracy improves gradually as the $L^{\theta}$ value increases up until 35 for Custom dataset. The experimental results revealed that the proposed face detection method might be the best for all the datasets for $L^{\theta}=35$.

Another experiment has been conducted for the important light intensity on some variety of images. Brighter images gradually darken lowering the $L^{a v}$ value and checked when Rule 1 with HFFD (i.e., HFFD + RGB) fails to detect face but Rule 2 with HFFD (i.e., HFFD + modified RGBR) can detect face. For darker images, the images were made lighten gradually and found out when Rule 1 start detecting faces. The output of this process is presented in Fig. 4 for a sample image. In the figure, the outcomes of standard HFFD are Red rectangles; Yellow rectangles are the outcome of Rule 1; and Green rectangles are the outcome of Rule 2. The average $L^{a v}$ in the detected region is shown to the top-left corner of each image. $L^{a v}$ value decreased from initial value of 62.7142 gradually. Both Rule 1 and Rule 2 can detect face while $L^{a v}>31.658$. For $L^{a v}<31.658$, Rule 1 cannot detect faces from images but Rule 2 still can detect face till $L^{a v}=2.5394$. Experiment shows that the proposed method can detect faces from low light images. It proves the efficiency of Rule 2 matching facial skin color in very low illumination condition which is even critical for human. Thus, it is an optimum choice for $L^{\theta}=35$ to apply Rule 1 and Rule 2 based on calculated $L^{a v}$ for above and below of $L^{\theta}$, respectively.

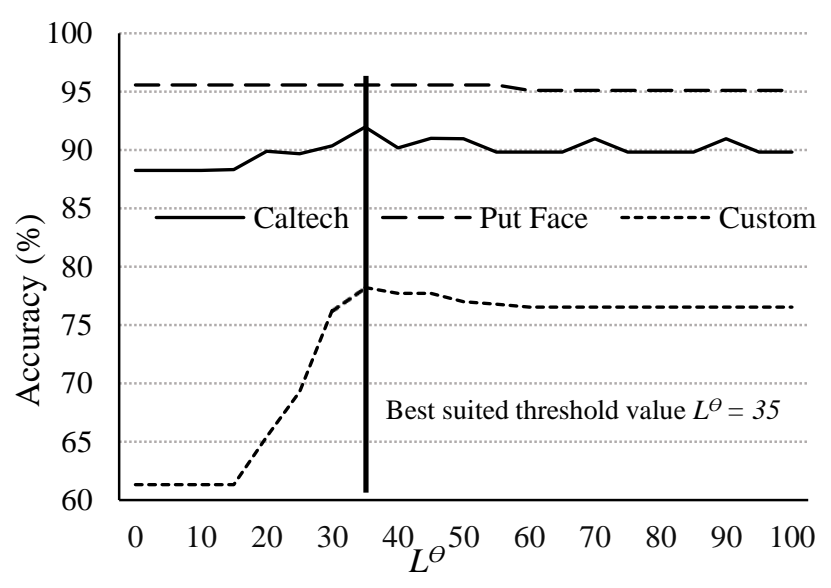

Fig. 3. Performance of the proposed method on images of Caltech, Put Face and Custom datasets with varying Light Intensity Threshold Value (i.e., $\left.L^{\theta}\right)$ 


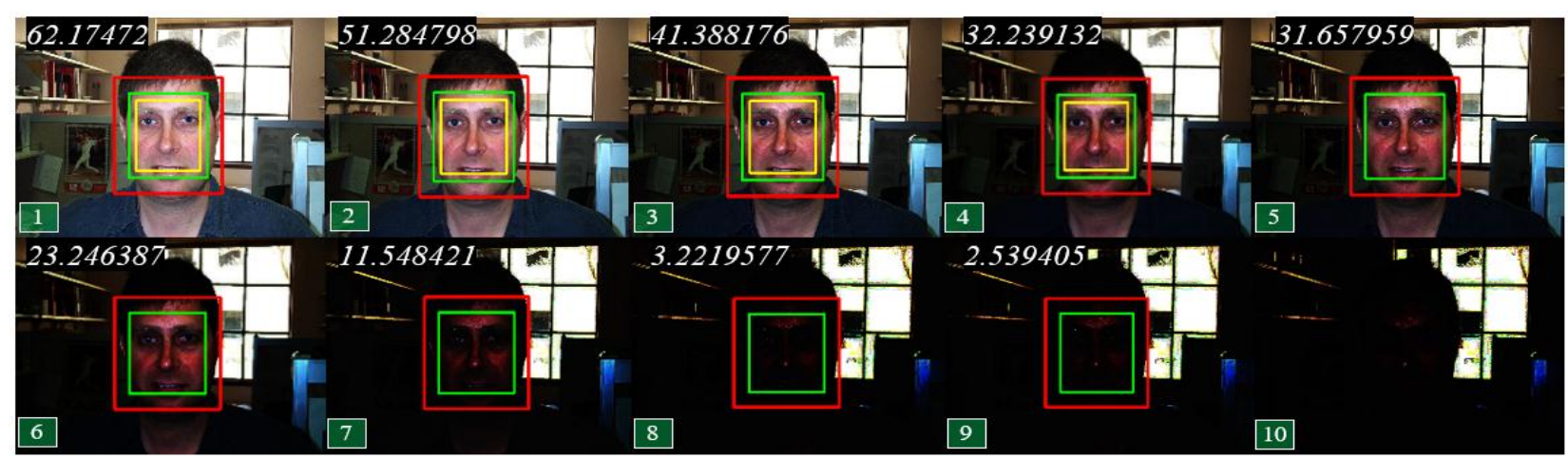

Fig. 4. Observation of face detection by Rule1 and Rule 2 of proposed methods on a sample image gradually darkening it; Red, Yellow and Green rectangles are the face detection by standard HFFD, Rule 1 and Rule 2, respectively. None of them can detect face when light intensity is less than 2.539405

\section{Demonstration of Proposed Face Detection Method}

A demonstration of face detection by the proposed method is presented in Fig. 5. In the first step, the original image Fig. 5(A) is passed through HAAR cascaded classifier of standard HFFD. The detected faces HFFD are marked by Red rectangles in Fig. 5(B); among seven detected faces, five are real faces (labeled as F1, F2, F3, F4 and F5) and two are false (labeled as F6 and F7). In the second step, the average light intensity of each individual face area is calculated and placed in the box. The average light intensities for F1, F2, F4 and F5 are above $L^{\theta}$. Therefore, Rule 1 was applied to skin color matching and all four satisfied Rule 1; thus, the faces are verified and marked by Yellow rectangles in Fig. 5(C). Again, the average light intensities of F3, F6 and F7 in Fig. 5(B) are below $L^{\theta}$. Therefore, Rule 2 was applied to filter them. But only F3 satisfied Rule 2 and therefore marked by Green rectangle in Fig.5(C); and F6 and F7 are identified as false faces (FP1 and FP2). Finally, the proposed system returned the faces which are verified by Rule 1(i.e., F1, F2, F4 and F5) or Rule 2 (i.e., F3).

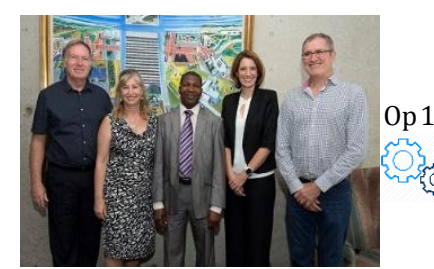

A. Original Image

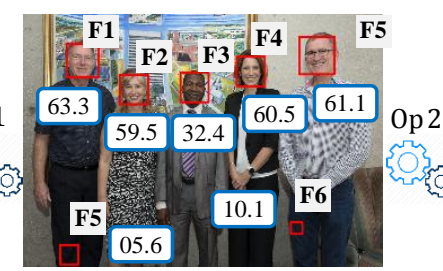

B. Primary Face Detected Image

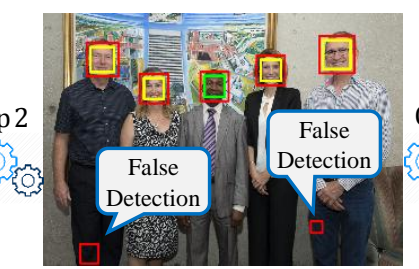

C. Color Filtered Image

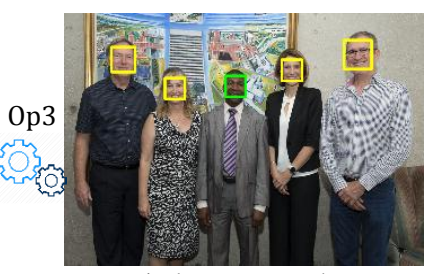

D. Final Face Detected Image

Fig. 5. Showing the demonstration of proposed face detection method step by steps. Red rectangles are the results of preliminary face detection by standard HFFD. Yellow and Green rectangles are the face verified by color filtering by Rule 1 and Rule 2, respectively. Op 1: Passing Through Haar Cascade Classifier; Op 2: Illumination Intensity Calculation and Applying Corresponding Color Filter; Op 3: Removing False Positives

\section{E. Experimental Results and Performance Analysis}

Experimental results and comparative performance analysis with two types of existing methods are presented: (I) methods with traditional human skin color detection and (II) methods with hybrid human skin color detection.

\section{I) Face Detection Methods with Traditional Human Skin Color Detection}

The three datasets used in the experiments are Caltech, Put Face and Custom datasets. The datasets contain different types of images for evaluations. Put Face dataset contains images of high resolution; Caltech dataset contains images of standard images; and Custom dataset contains images of dark-skinned face under lowlight conditions. In addition, a dataset is prepared combining the three and the results are compared with it. Detail results are presents in Table 1 where the performance measurement is presented separately for each dataset. The sample of resulting images of each dataset is presented in Fig. 6. Four sample images are presented from each of Caltech and Put Face datasets and one sample image from Custom dataset as it contains many faces. Red color presents standard HFFD. Yellow, Orange, Blue and Green color represent HFFD + RGB, HFFD + NRGB, HFFD + YCBCR, HFFD + RGBR, respectively. Purple color is used to represent the proposed method. 
Table 1. Performance Comparison (\%) of Proposed Method with Existing Methods

\begin{tabular}{|c|c|c|c|c|c|}
\hline Dataset & Method & $\mathbf{T P}$ & FP & TN & Acc. \\
\hline \multirow{6}{*}{ Caltech } & Standard HFFD & 98.07 & 15.02 & 1.90 & 85.26 \\
\hline & HFFD + RGB & 90.99 & 2.57 & 9.01 & 88.70 \\
\hline & HFFD + NRGB & 64.38 & 1.29 & 35.62 & 86.44 \\
\hline & $\mathrm{HFFD}+\mathrm{YCbCr}$ & 87.55 & 2.36 & 12.45 & 85.53 \\
\hline & HFFD + RGBR & 68.88 & 4.08 & 31.12 & 66.19 \\
\hline & Proposed Method & 93.35 & 3.86 & 6.65 & 89.88 \\
\hline \multirow{6}{*}{ Put Face } & HFFD & 98.05 & 23.35 & 1.95 & 79.50 \\
\hline & HFFD + RGB & 98.05 & 2.63 & 1.95 & 95.55 \\
\hline & HFFD-N_RGB & 97.67 & 3.02 & 2.33 & 94.81 \\
\hline & $\mathrm{HFFD}+\mathrm{YCbCr}$ & 98.05 & 2.33 & 1.95 & 95.81 \\
\hline & HFFD + RGBR & 98.05 & 3.11 & 1.95 & 95.09 \\
\hline & Proposed Method & 98.05 & 2.63 & 1.95 & 95.55 \\
\hline \multirow{6}{*}{ Custom } & HFFD & 87.74 & 11.61 & 12.26 & 78.61 \\
\hline & HFFD + RGB & 62.90 & 1.94 & 37.10 & 61.32 \\
\hline & HFFD-N_RGB & 55.50 & 0.97 & 44.50 & 54.95 \\
\hline & $\mathrm{HFFD}+\mathrm{YCbCr}$ & 60.00 & 2.26 & 40 & 58.68 \\
\hline & HFFD + RGBR & 76.45 & 2.58 & 23.55 & 74.53 \\
\hline & Proposed Method & 87.74 & 4.19 & 12.26 & 84.21 \\
\hline \multirow{6}{*}{ Combined } & HFFD & 96.28 & 20.84 & 4.82 & 78.96 \\
\hline & HFFD + RGB & 90.08 & 2.49 & 9.80 & 87.99 \\
\hline & HFFD-N_RGB & 81.87 & 2.21 & 18.18 & 80.06 \\
\hline & $\mathrm{HFFD}+\mathrm{YCbCr}$ & 88.80 & 2.32 & 11.19 & 86.80 \\
\hline & HFFD + RGBR & 86.80 & 3.27 & 13.19 & 84.06 \\
\hline & Proposed Method & 95.06 & 3.38 & 4.93 & 91.96 \\
\hline
\end{tabular}

Caltech Dataset: Although standard HFFD showed good TP value for Caltech dataset but overall performance decreased due to high FP value. Standard HFFD detected 457 faces correctly from 466 and made 70 false positives. Therefore, standard HFFD achieved an accuracy of $85.26 \%$. Any other method showed less FP value and but higher TN value. The proposed method detected 435 faces correctly with only 18 false positives and 31 true negatives; the achieved accuracy is $89.88 \%$. For the dataset, HFFD + RGB was a close competitor of the proposed method showing the accuracy of $88.70 \%$.

Put Face Dataset: The images of this dataset are very clean and the illumination condition is very good. Therefore, all the methods performed very well except standard HFFD which has a lot of false positives. For the dataset, HFFD + YCBCR, HFFD + RGBR and the proposed method achieved the same TP (98.05\%) and TN (1.95\%) values. However, the accuracy of HFFD + YCbCr is little better than the proposed method due to little better performance in FP.

Custom Dataset: Face detection from custom dataset is more challenging. Table 1 shows that the proposed method is much better than others on this dataset. We found that all of the existing color matching methods suffer from dark-skinned face and low light condition. The proposed method has the same TP $(87.74 \%)$ of the standard HFFD but it minimized FP significantly. Other methods have much lower TP with higher TN. Finally, the proposed method achieved an accuracy of $84.21 \%$, which is higher than any other method.

Combined Dataset: Overall performance of these six methods is also measured against the combined Caltech, Put Face and Custom datasets. The combined dataset contains total of 1806 faces. Among the existing methods, HFFD has better TP (i.e., 96.28\%) and TN (i.e., 4.82\%) but suffers from very high FP (i.e., 20.84\%). HFFD showed an accuracy of $78.96 \%$. Other exiting methods significantly minimized FP but they have high TN and low TP. HFFD + RGB is the best among the existing methods showing an accuracy of $87.99 \%$. On the other hand, the proposed method showed TP of $95.06 \%$ (truly detecting 1715 faces), FP of $3.38 \%$ and TN of $4.93 \%$. The performance of the proposed method was verified on the individual datasets, which showed an accuracy of $91.96 \%$ on the combined dataset. 


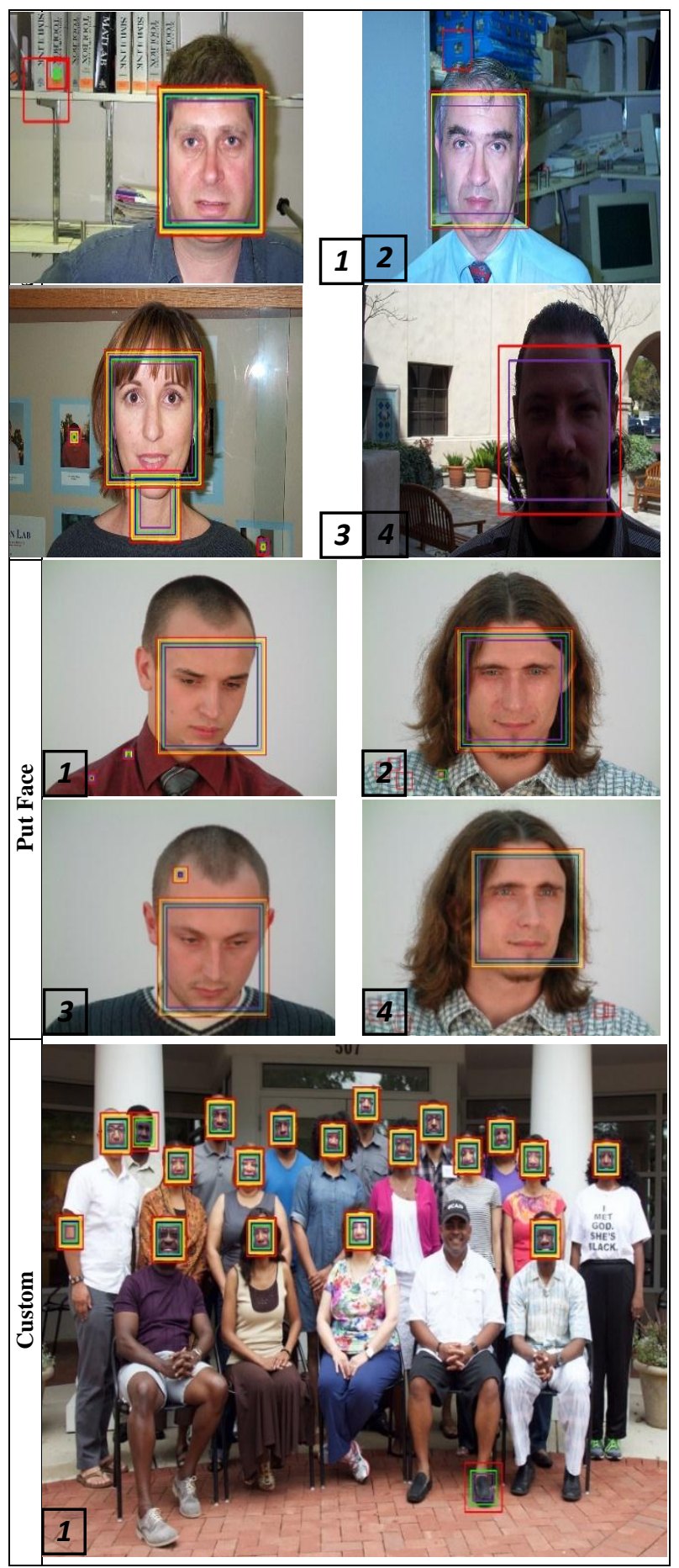

Fig. 6. Results on nine sample images from Caltech, Put Face and Custom datasets. Red rectanges represents standard HFFD. Yellow, Orange, Blue and Green rectangle represent HFFD + RGB, HFFD + NRGB, HFFD + YCBCR and HFFD + RGBR, respectively. Purple ractengle is for the proposed method.

\section{Overall Observation:}

HFFD + RGB performed better for Caltech dataset which contains standard type of images but suffer from detecting face from Custom dataset containing images of dark-skinned faces and images with low illumination condition. On the other hand, HFFD + RGBR performed much better than HFFD + RGB on the Custom dataset but achieved lower accuracy than HFFD + RGB for Caltech dataset. Moreover, the standard HFFD performed better than the other traditional methods on Custom dataset but achieved much lower accuracy than other methods for Caltech and Put datasets. It leads to conclusion that there is no single method that can win over all the datasets. Interestingly, the proposed method showed the best accuracy for all the three datasets. 


\section{I) Face Detection Methods with Hybrid Human Skin Color Detection}

This section compares the performance of the proposed method with several existing face detection methods with hybrid skin color matching. Fresh experiments were conducted on Bao, Muct and Caltech datasets as there are several exiting studies available on the datasets with hybrid skin color matching. Outcomes of different methods are presented separately for each dataset in Table 2. Method 1 (RGB+ YCbCr)[51] is implemented in this study. Results of Method 2 (Red + HSL)[52] and Method 3 (RGB + YCbCr +HSV)[53] are the reported results in the papers where the sign '-' indicates no- availability of results. The performance of the methods on the individual datasets are discussed below.

Bao Dataset: There are total of 1206 faces in the multiple face sets in Bao dataset. Different methods are considered for individual face types. The proposed method has been tested for three different face types for proper comparison. Method 1 showed low FP for any face types and the achieved accuracies are 88.62\%, 91.70\% and 96.72\% for all types of faces, multiple frontal faces and single frontal faces, respectively. For this dataset, Method 3 is carried out on selected 157 images of all type faces and achieved an accuracy of $92.41 \%$. The proposed method showed competitive performance compared to Method 3 for all type of faces; although the comparison with Method 3 is not completely justified due to the difference in image numbers. On the other hand, frontal face cases, the proposed method outperformed Method 1 significantly showing accuracies of $94.65 \%$ and $97.54 \%$ for multiple frontal faces and single frontal faces, respectively. The standard HFFD is used as the preliminary face detector for the frontal face features. Therefore, it is likely that the proposed method will outperform for frontal faces.

Muct Dataset: The images of this dataset are distorted by artificial light. Among 751 images, 27 images are extremely distorted and do not contain skin color properties at all. Two experiments were carried out on the dataset with and without distorted images. For the dataset, Method 1 achieved the accuracies of $81.04 \%$ and $83.65 \%$ for all the faces including distorted image and natural color images excluding 27 distorted images, respectively. Method 2 and Method 3 are applied to all faces only and achieved the accuracies of $80.17 \%$ and $97.27 \%$, respectively. The proposed method showed higher accuracy over Method 1 for natural color images and achieved an accuracy of $97.17 \%$. On the other, the proposed method achieved an accuracy of $93.80 \%$ for all faces. The achieved accuracy is much higher than Method $1 /$ Method 2 and competitive to Method 3.

Caltech Dataset: There are 450 images containing 466 faces in the dataset. For this dataset, Method 1 and Method 2 showed an accuracy of $85.53 \%$ and $88.57 \%$ respectively. On the other hand, the proposed method outperformed both the methods showing an accuracy of $89.90 \%$.

\section{Overall Observation:}

The performance measurements show that the proposed method is better than Method 1 and Method 2 for Bio dataset in all aspects. For Muct and Caltech datasets, the proposed method outperformed Method 1 and Method 2. Method 3 is relatively complex combining three individual measures. However, the proposed method showed higher accuracy than Method 3 in the case of frontal faces and single face containing set of Bao dataset.

\section{Conclusions}

In this study, a robust face detection is investigated integrating a novel skin color matching method. The proposed skin color matching method is an illumination based generalized method combining two different methods which are individually good for high and low illumination conditions, respectively. The proposed method is incorporated with HFFD to reduce false detections and is found remarkable results working properly on wide range of illumination conditions. Experimental results show that the proposed face detection technique is suitable for detecting face from almost all kind of skin color and illumination conditions outperforming the existing methods. The proposed skin color detection technique might be suitable to improve other skin color matching issues such as detecting and tracking of different body parts and remains as a future study.

\section{References}

[1] A. M. Asif Anjum Akash, Abdus Salim Mollah and M. A. H, Akhand, "Improvement of Haar Feature Based Face Detection in OpenCV Incorporating Human Skin Color Characteristic,” J. Comput. Sci. Appl. Inf. Technol., vol. 1, no. 1, pp. 1-8, 2016, doi: 10.15226/2474-9257/1/1/00106.

[2] R. Ranjan et al., "A Fast and Accurate System for Face Detection, Identification, and Verification," IEEE Trans. Biometrics, Behav. Identity Sci., vol. 1, no. 2, pp. 82-96, 2019, doi: 10.1109/tbiom.2019.2908436.

[3] S. S. Rajput and K. V. Arya, "Noise Robust Face Hallucination via Outlier Regularized Least Square and Neighbor Representation," IEEE Trans. Biometrics, Behav. Identity Sci., vol. PP, no. XX, pp. 1-1, 2019, doi: 10.1109/tbiom.2019.2939808.

[4] Z. Zakaria, S. A. Suandi, and J. Mohamad-Saleh, "Hierarchical Skin-AdaBoost-Neural Network (H-SKANN) for multi-face detection," Appl. Soft Comput., vol. 68, pp. 172-190, Jul. 2018, doi: 10.1016/j.asoc.2018.03.030.

[5] R. Féraud, O. J. Bernier, J. E. Viallet, and M. Collobert, "A fast and accurate face detector based on neural networks," IEEE 
Trans. Pattern Anal. Mach. Intell., 2001, doi: 10.1109/34.899945.

[6] L.-L. Huang, A. Shimizu, Y. Hagihara, and H. Kobatake, "Face detection from cluttered images using a polynomial neural network," Neurocomputing, vol. 51, pp. 197-211, Apr. 2003, doi: 10.1016/S0925-2312(02)00616-1.

[7] K. Zhang, Z. Zhang, Z. Li, and Y. Qiao, "Joint Face Detection and Alignment Using Multitask Cascaded Convolutional Networks,” IEEE Signal Process. Lett., vol. 23, no. 10, pp. 1499-1503, Oct. 2016, doi: 10.1109/LSP.2016.2603342.

[8] D. Triantafyllidou, P. Nousi, and A. Tefas, "Fast Deep Convolutional Face Detection in the Wild Exploiting Hard Sample Mining,” Big Data Res., vol. 11, pp. 65-76, Mar. 2018, doi: 10.1016/j.bdr.2017.06.002.

[9] W. T. Chu and W. W. Li, "Manga face detection based on deep neural networks fusing global and local information," Pattern Recognit., 2019, doi: 10.1016/j.patcog.2018.08.008.

[10] X. Sun, P. Wu, and S. C. H. Hoi, "Face detection using deep learning: An improved faster RCNN approach," Neurocomputing, vol. 299, pp. 42-50, Jul. 2018, doi: 10.1016/j.neucom.2018.03.030.

[11] B. Heisele, T. Serre, S. Prentice, and T. Poggio, "Hierarchical classification and feature reduction for fast face detection with support vector machines," Pattern Recognit., vol. 36, no. 9, pp. 2007-2017, Sep. 2003, doi: 10.1016/S0031-3203(03)00062-1.

[12]H. Pan, Y. Zhu, and L. Xia, "Efficient and accurate face detection using heterogeneous feature descriptors and feature selection," Comput. Vis. Image Underst., vol. 117, no. 1, pp. 12-28, Jan. 2013, doi: 10.1016/j.cviu.2012.09.003.

[13] Z. Jin, Z. Lou, J. Yang, and Q. Sun, "Face detection using template matching and skin-color information," Neurocomputing, vol. 70, no. 4-6, pp. 794-800, Jan. 2007, doi: 10.1016/j.neucom.2006.10.043.

[14] J. Miao, B. Yin, K. Wang, L. Shen, and X. Chen, "A hierarchical multiscale and multiangle system for human face detection in a complex background using gravity-center template," Pattern Recognit., vol. 32, no. 7, pp. 1237-1248, Jul. 1999, doi: 10.1016/S0031-3203(98)00156-3.

[15] M. R. Mahmoodi and S. M. Sayedi, "A face detection method based on kernel probability map," Comput. Electr. Eng., vol. 46, pp. 205-216, Aug. 2015, doi: 10.1016/j.compeleceng. 2015.02.005.

[16] K. K. Sung and T. Poggio, "Example-based learning for view-based human face detection," IEEE Trans. Pattern Anal. Mach. Intell., 1998, doi: 10.1109/34.655648.

[17] A. Atharifard and S. Ghofrani, "Robust Component-based Face Detection Using Color Feature," Proc. World Congr. Eng., vol. 2, pp. 6-8, 2011.

[18] F. Jiang, M. Fischer, H. K. Ekenel, and B. E. Shi, "Combining texture and stereo disparity cues for real-time face detection," Signal Process. Image Commun., vol. 28, no. 9, pp. 1100-1113, Oct. 2013, doi: 10.1016/j.image.2013.07.006.

[19] Y. H. Tsai, Y. C. Lee, J. J. Ding, R. Y. Chang, and M. C. Hsu, "Robust in-plane and out-of-plane face detection algorithm using frontal face detector and symmetry extension," Image Vis. Comput., 2018, doi: 10.1016/j.imavis.2018.07.003.

[20] K. W. Wong, K. M. Lam, and W. C. Siu, "A robust scheme for live detection of human faces in color images," Signal Process. Image Commun., 2003, doi: 10.1016/S0923-5965(02)00088-7.

[21] J. Yan, X. Zhang, Z. Lei, and S. Z. Li, "Face detection by structural models," Image Vis. Comput., vol. 32, no. 10, pp. 790-799, Oct. 2014, doi: 10.1016/j.imavis.2013.12.004.

[22] P. Viola and M. Jones, "Rapid object detection using a boosted cascade of simple features," in Proceedings of the 2001 IEEE Computer Society Conference on Computer Vision and Pattern Recognition. CVPR 2001, vol. 1, pp. I-511-I-518, doi: 10.1109/CVPR.2001.990517.

[23] M. Yang, J. Crenshaw, B. Augustine, R. Mareachen, and Y. Wu, "AdaBoost-based face detection for embedded systems," Comput. Vis. Image Underst., vol. 114, no. 11, pp. 1116-1125, Nov. 2010, doi: 10.1016/j.cviu.2010.03.010.

[24] D. Chen, S. Ren, Y. Wei, X. Cao, and J. Sun, "Joint Cascade Face Detection and Alignment," in Lecture Notes in Computer Science (including subseries Lecture Notes in Artificial Intelligence and Lecture Notes in Bioinformatics), 2014, pp. 109-122.

[25] F. Faux and F. Luthon, "Theory of evidence for face detection and tracking," Int. J. Approx. Reason., vol. 53, no. 5, pp. 728-746, Jul. 2012, doi: 10.1016/j.ijar.2012.02.002.

[26] S. Liao, A. K. Jain, and S. Z. Li, "A Fast and Accurate Unconstrained Face Detector," IEEE Trans. Pattern Anal. Mach. Intell., vol. 38, no. 2, pp. 211-223, Feb. 2016, doi: 10.1109/TPAMI.2015.2448075.

[27] G. Hemalatha and C. P. Sumathi, “A Study of Techniques for Facial Detection and,” vol. 5, no. 2, pp. 27-37, 2014.

[28] V. Vezhnevets, V. Sazonov, and A. Andreeva, "A Survey on Pixel-Based Skin Color Detection Techniques," Cybernetics, 2003, doi: R. Khan, A. Hanbury, J. Stöttinger, and A. Bais, "Color based skin classification," Pattern Recognition Letters, vol. 33, no. 2, pp. 157-163, Jan. 2012.

[29] R. Belaroussi and M. Milgram, "A comparative study on face detection and tracking algorithms," Expert Syst. Appl., vol. 39, no. 8, pp. 7158-7164, Jun. 2012, doi: 10.1016/j.eswa.2012.01.076.

[30] E. Hjelmås and B. K. Low, "Face detection: A survey," Comput. Vis. Image Underst., vol. 83, no. 3, pp. 236-274, 2001, doi: 10.1006/cviu.2001.0921.

[31] H. A. Rowley, S. Baluja, and T. Kanade, "Neural network-based face detection," IEEE Trans. Pattern Anal. Mach. Intell., vol. 20, no. 1, pp. 23-38, 1998, doi: 10.1109/34.655647.

[32] S. Kang and S. Park, "A fusion neural network classifier for image classification," Pattern Recognit. Lett., vol. 30, no. 9, pp. 789-793, Jul. 2009, doi: 10.1016/j.patrec.2008.06.009.

[33] M. Bianchini, M. Maggini, L. Sarti, and F. Scarselli, "Recursive neural networks learn to localize faces," Pattern Recognit. Lett., vol. 26, no. 12, pp. 1885-1895, Sep. 2005, doi: 10.1016/j.patrec.2005.03.010.

[34] H. M. El-Bakry, "Face detection using fast neural networks and image decomposition," Neurocomputing, vol. 48, no. 1-4, pp. 1039-1046, Oct. 2002, doi: 10.1016/S0925-2312(02)00608-2.

[35] H. C. V. Lakshmi and S. PatilKulakarni, "Segmentation Algorithm for Multiple Face Detection in Color Images with Skin Tone Regions using Color Spaces and Edge Detection Techniques," Int. J. Comput. Theory Eng., pp. 552-558, 2010, doi: 10.7763/IJCTE.2010.V2.200.

[36] P. Kakumanu, S. Makrogiannis, and N. Bourbakis, "A survey of skin-color modeling and detection methods," Pattern Recognit., vol. 40, no. 3, pp. 1106-1122, Mar. 2007, doi: 10.1016/j.patcog.2006.06.010.

[37] C. Lin, "Face detection in complicated backgrounds and different illumination conditions by using YCbCr color space and 
neural network," Pattern Recognit. Lett., vol. 28, no. 16, pp. 2190-2200, Dec. 2007, doi: 10.1016/j.patrec.2007.07.003.

[38] Y. Ban, S.-K. Kim, S. Kim, K.-A. Toh, and S. Lee, "Face detection based on skin color likelihood," Pattern Recognit., vol. 47, no. 4, pp. 1573-1585, Apr. 2014, doi: 10.1016/j.patcog.2013.11.005.

[39] F. Solina, P. Peer, B. Batagelj, S. Juvan, and J. Kovac, "Color-based Face Detection in the ' 15 Seconds of Fame' Art Installation,” Art Install., 2003, doi: 10.1016/J.CATTOD.2013.08.026.

[40] Zhengming Li, Lijie Xue, and Fei Tan, "Face detection in complex background based on skin color features and improved AdaBoost algorithms," in 2010 IEEE International Conference on Progress in Informatics and Computing, 2010, pp. 723-727, doi: 10.1109/PIC.2010.5687939.

[41] J. M. Chaves-González, M. A. Vega-Rodríguez, J. A. Gómez-Pulido, and J. M. Sánchez-Pérez, "Detecting skin in face recognition systems: A colour spaces study," Digit. Signal Process. A Rev. J., vol. 20, no. 3, pp. 806-823, May 2010, doi: 10.1016/j.dsp.2009.10.008

[42] N. A. bin Abdul Rahman, K. C. Wei, and J. See, "RGB-H-CbCr Skin Colour Model for Human Face Detection," Proc. MMU Int. Symp. Inf. Commun. Technol. (M2USIC 2006), 2006.

[43] H. M. Z. Hewa Majeed Zangana, “A New Algorithm for Human Face Detection Using Skin Color Tone," IOSR J. Comput. Eng., vol. 11, no. 6, pp. 31-38, 2013, doi: 10.9790/0661-1163138.

[44] A. Mohamed, Y. Weng, J. Jiang, and S. Ipson, "Face detection based neural networks using robust skin color segmentation," in 2008 5th International Multi-Conference on Systems, Signals and Devices, 2008, pp. 1-5, doi: 10.1109/SSD.2008.4632827.

[45] A. Cheddad, J. Condell, K. Curran, and P. Mc Kevitt, "A skin tone detection algorithm for an adaptive approach to steganography," Signal Processing, vol. 89, no. 12, pp. 2465-2478, Dec. 2009, doi: 10.1016/j.sigpro.2009.04.022.

[46] D. Ghimire and J. Lee, "A Robust Face Detection Method Based on Skin Color and Edges," J. Inf. Process. Syst., vol. 9, no. 1, pp. 141-156, Mar. 2013, doi: 10.3745/JIPS.2013.9.1.141.

[47] Y. J. Fu and J. W. Li, "Rotation invariant multi-view color face detection based on skin color and Adaboost algorithm," in 2010 International Conference on Biomedical Engineering and Computer Science, ICBECS 2010, 2010, doi: 10.1109/ICBECS.2010.5462517.

[48] M. A. A. Akash, M. A. H. Akhand, and N. Siddique, "Robust Face Detection Using Hybrid Skin Color Matching under Different Illuminations," in 2019 International Conference on Electrical, Computer and Communication Engineering (ECCE), 2019, pp. 1-6, doi: 10.1109/ECACE.2019.8679481.

[49] J. Kova??, P. Peer, and F. Solina, "Human skin colour clustering for face detection," in IEEE Region 8 EUROCON 2003. Computer as a Tool - Proceedings, 2003, doi: 10.1109/EURCON.2003.1248169.

[50] G. Gomez and E. F. Morales, "Automatic Feature Construction and a Simple Rule Induction Algorithm for Skin Detection," Work. Mach. Learn. Comput. Vis., no. May, pp. 31-38, 2002.

[51] G. Kukharev and A. Nowosielski, "Visitor Identification - Elaborating Real Time Face Recognition System," WSCG (Short Pap., no. January 2004, pp. 157-164, 2004.

[52] M. A. Berbar, "Skin colour correction and faces detection techniques based on HSL and R colour components," Int. J. Signal Imaging Syst. Eng., vol. 7, no. 2, pp. 104-115, 2014, doi: 10.1504/IJSISE.2014.060056.

[53] S. Yadav and N. Nain, “A novel approach for face detection using hybrid skin color model,” J. Reliab. Intell. Environ., vol. 2, no. 3, pp. 145-158, 2016, doi: 10.1007/s40860-016-0024-8.

[54]V. S. Bhat and J. D. Pujari, “A Hybrid Skin Color Model for Face Detection,” Int. J. Eng. Res. Gen. Sci., vol. 2, no. 2, pp. 68-75, 2014.

[55] "Illumination Intensity Calculation Formula," 2019. [Online]. Available: http://www.whatafuture.com/lg-ac-with-ionizer-thathas-human-detection-sensors/.

[56] “Accuracy calculation formula.”[Online]. Available: https://en.wikipedia.org/wiki/Sensitivity_and_specificity. [Accessed: 29Oct-2019].

[57] "Caltech dataset," 2019. [Online]. Available: http://www.vision.caltech.edu/archive.html. [Accessed: 29-Oct-2019].

[58] "Put face database," 2019. [Online]. Available: https://biometrics.cie.put.poznan.pl/index.php?view=article\&id=4. [Accessed: 29-Oct-2019].

[59] "Bao database." [Online]. Available: https://facedetection.com/datasets/. [Accessed: 29-Oct-2019].

[60] "Muct database available:" [Online]. Available: http://www.milbo.org/muct/. [Accessed: 29-Oct-2019].

\section{Authors' Profiles}

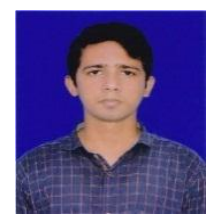

Asif Anjum Akash received B.Sc. and M.Sc. degrees in Computer Science and Engineering (CSE) from Khulna University of Engineering and Technology (KUET), Bangladesh in 2015 and 2019, respectively. After graduation he joined in the Dept. of CSE, North Western University, Khulna as a Lecturer. At present, he is serving as an Assistant Programmer in KUET. His research interest includes pattern recognition, image processing and advanced machine learning.

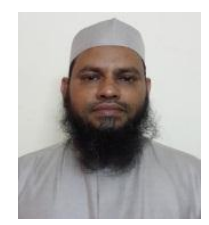

M. A. H. Akhand received his B.Sc. degree in Electrical and Electronic Engineering from Khulna University of Engineering and Technology (KUET), Bangladesh in 1999, the M.E. degree in Human and Artificial Intelligent Systems in 2006, and the Ph.D. degree in System Design Engineering in 2009 from University of Fukui, Japan. He joined as a lecturer at the Department of Computer Science and Engineering at KUET in 2001, and is now a Professor. He is also head of Computational Intelligence Research Group of this department. He is a member of Institution of Engineers, Bangladesh (IEB) and a life time member Bangladesh Computer Society (BCS). He has 
more than 100 research publications. His research interest includes artificial neural networks, evolutionary computation, bioinformatics, swarm intelligence and other bio-inspired computing techniques. Dr. Akhand received several best paper Prizes in international conferences.

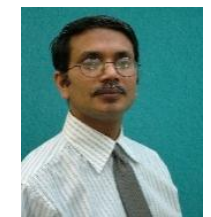

N. Siddique is with the School of Computing, Engineering and Intelligent Systems, Ulster University. He obtained Dipl.-Ing. degree in Cybernetics from the TU Dresden, Germany, MSc in Computer Science from BUET, Bangladesh and $\mathrm{PhD}$ in Intelligent Control from the Department of Automatic Control and Systems Engineering, University of Sheffield, England. His research interests include: robotics, cybernetics, computational intelligence, nature-inspired computing, stochastic systems and vehicular communication. He has published over 170 research papers. He authored and co-authored five books published by John Wiley, Springer and Taylor \& Francis. He guest edited eight special issues of reputed journals on Cybernetic Intelligence, Computational Intelligence, Neural Networks and Robotics. He has been involved in organizing many international conferences and co-edited conference proceedings. He is a Fellow of the Higher Education Academy, a senior member of IEEE and a member of different committees of IEEE SMCS. He is on the editorial board of the Nature Scientific Research, Journal of Behavioural Robotics, Engineering Letters, International Journal of Machine Learning and Cybernetics, International Journal of Applied Pattern Recognition, International Journal of Advances in Robotics Research and also on the editorial advisory board of the International Journal of Neural Systems.

How to cite this paper: Asif Anjum Akash, M. A. H. Akhand, N. Siddique, " Robust Face Detection integrating Novel Skin Color Matching under Variant Illumination Conditions", International Journal of Image, Graphics and Signal Processing(IJIGSP), Vol.13, No.2, pp. 1-15, 2021.DOI: 10.5815/ijigsp.2021.02.01 